\title{
Geodesic flows on Riemannian g.o. spaces*
}

\author{
Božidar Jovanović \\ Mathematical Institute SANU, Serbian Academy of Sciences and Arts ${ }^{\dagger}$
}

\begin{abstract}
We prove the integrability of geodesic flows on the Riemannian g.o. spaces of compact Lie groups, as well as on a related class of Riemannian homogeneous spaces having an additional principal bundle structure 1
\end{abstract}

\section{Introduction}

1.1. Let $\left(Q, d s^{2}\right)$ be a Riemannian manifold. A geodesic in $Q$ is called homogeneous if it is the orbit of an one-parameter group of isometries of $Q$. A Riemannian homogeneous space $\left(Q=G / H, d s^{2}\right)$ is a g.o. (geodesic orbit) space with respect to $G$ if every geodesic is the orbit of an one-parameter subgroup of $G$. Every naturally reductive space, in particular a homogeneous space $G / H$ with a normal metric $d s_{0}^{2}$, is a g.o. space. The converse is not true and the first counter example was found by Kaplan [19], which motivated the study of g.o. spaces, e.g., see [20, 15, 6, 21, 13, 34, 1, 2].

In Lagrangian and Hamiltonian mechanics homogeneous geodesics are related to the notion of relative equilibria, e.g, see 22, 36 and references therein. Following the line of Hamiltonian mechanics, in this paper we study geodesic flows on g.o. spaces $\left(Q=G / H, d s^{2}\right)$ of compact Lie groups $G$. Since the geodesics are orbits, it is naturally to expect that the corresponding geodesic flows, as in the case of normal metrics [8, 9, 17, are completely integrable. Indeed, we prove their integrability in a noncommutative sense [29, 24, 23].

1.2. Let $\left(\mathcal{F}_{G},\{\cdot, \cdot\}\right)$ be the algebra of $G$-invariant analytic, polynomial in momenta functions on $T^{*}(G / H)$, where $T^{*} Q$ is endowed with the canonical Poisson bracket. The Hamiltonian of a normal metric $d s_{0}^{2}$ is a central function within the algebra $\left(\mathcal{F}_{G},\{\cdot, \cdot\}\right)$. The main observation is that this property can be used for a characterization of g.o. metrics on $G / H$ : the Hamiltonian of a $G$-invariant metric $d s^{2}$ is a central function in $\mathcal{F}_{G}$ if and only if $d s^{2}$ is a g.o. metric (Lemma 2, Section 2).

Now, the integrability of geodesic flows on g.o. spaces follows from a general geometric construction given in [8, 9]. Let $\Phi: T^{*} Q \rightarrow \mathfrak{g}^{*}$ be the momentum mapping of the natural $G$-action. According to the Noether theorem, the momentum mapping $\Phi$ is conserved along the flows of $G$-invariant Hamiltonians: $\left\{\mathcal{F}_{G}, \Phi^{*}(\mathbb{R}[\mathfrak{g}])\right\}=0$. Moreover, the collection of functions

$$
\mathcal{F}=\mathcal{F}_{G}+\Phi^{*}\left(\mathbb{R}\left[\mathfrak{g}^{*}\right]\right)
$$

*Journal ref: Regular and Chaotic Dynamics 16 (2011), No. 5, 504-513 - issue: the conference Geometry, Dynamics, Integrable Systems GDIS 2010 (Serbia, September 7-13, 2010) dedicated to the 60th birthdays of B. A. Dubrovin, V. V. Kozlov, I. M. Krichever, and A. I. Neishtadt

${ }^{\dagger}$ Kneza Mihaila 36, 11000 Belgrade, Serbia, e-mail: bozaj@mi.sanu.ac.rs

${ }^{1}$ MSC: 70H06, 37J35, 53D25 
is a complete set of functions on $T^{*} Q$ :

$$
\operatorname{ddim} \mathcal{F}+\operatorname{din} d \mathcal{F}=\operatorname{dim} T^{*} Q .
$$

Here, for a set of functions $\mathcal{F}$ closed under the Poisson bracket, such that the dimensions of $\mathrm{F}_{x}=\langle d f(x) \mid f \in \mathcal{F}\rangle$ and $\left.\operatorname{ker}\{\cdot, \cdot\}\right|_{\mathrm{F}_{x} \times \mathrm{F}_{x}}$ are constant on an open dense set $U \subset M$, we denote $\operatorname{ddim} \mathcal{F}=\operatorname{dim} \mathrm{F}_{x}$ and dind $\mathcal{F}=\left.\operatorname{dim} \operatorname{ker}\{\cdot, \cdot\}\right|_{\mathrm{F}_{x} \times \mathrm{F}_{x}}, x \in U$ (differential dimension and differential index of $\mathcal{F})$ 9, 17 .

Since central functions in $\mathcal{F}_{G}$ Poisson commute both with $\mathcal{F}_{G}$ and $\Phi^{*}\left(\mathbb{R}\left[\mathfrak{g}^{*}\right]\right)$, we get:

Theorem 1: The geodesic flow of a g.o. space $\left(Q=G / H, d s^{2}\right)$ is completely integrable with a complete noncommutative set of integrals (1).

1.3. Mishchenko and Fomenko stated the conjecture that noncommutative integrability implies the usual Liouville one by means of integrals that belong to the same functional class as noncommutative integrals [24, 31, 9]. One can always construct a complete commutative subset within $\Phi^{*}\left(\mathfrak{g}^{*}\right)$. Thus, for a complete set (1), the conjecture reduces to a construction of a complete commutative subset $\mathcal{B} \subset \mathcal{F}_{G}$ (see [9, 10] )

$$
\operatorname{ddim} \mathcal{B}=\operatorname{dind} \mathcal{B}=\frac{1}{2}\left(\operatorname{ddim} \mathcal{F}_{G}+\operatorname{dind} \mathcal{F}_{G}\right) .
$$

If the required subset $\mathcal{B} \subset \mathcal{F}_{G}$ exist, we say that $(G, H)$ is an integrable pair. There are several known classes of integrable pairs (see [35, 25, 27, 8, 10, 26, 12, 18]) but the general problem rest still unsolved.

We recall the known examples of g.o. spaces $G / H$ with non-normal metrics of compact Lie groups (Section 2). In most of them, the underlying metrics are deformations $d s_{\lambda}^{2}$ of a normal metric in the fiber direction of the bundle

$$
K / H \longrightarrow Q=G / H \longrightarrow M=G / K,
$$

where $K$ is a subgroup of $G, H \subset K \subset G$.

Tamaru classified triples $(G, K, H)$ where $(G, K)$ is a compact effective irreducible symmetric pair and $\left(G / H, d s_{\lambda}^{2}\right)$ is a g.o. space [34]. It turns out that these homogeneous spaces have the complexity equal 0 or 1 (see the classification of complexity 0 and 1 homogeneous spaces of the reductive Lie groups [25, 38, 30, 27, 44). This implies, by the result of Mykytyuk and Stepin [27, that the appropriate geodesic flows are Liouville integrable by means of analytic functions, polynomial in momenta (Theorem 4). For a reader's sake, by analyzing the algebra of $G$-invariant functions $\mathcal{F}_{G}$ a direct derivation of the complexity and commuting integrals for various g.o. spaces is given (Theorem 4, Examples 4-7, Section $4)$.

As a bi-product, in Section 3 we obtain a class of Riemannian homogeneous spaces, defined by fibration (44), with completely integrable geodesic flows (Theorem 3, Examples 1 - 3). In particular, we have (see Example 1):

Theorem 2: The homogeneous spaces fibered over irreducible symmetric spaces with invariant Einstein metrics classified by Jensen [16] have completely integrable geodesic flows.

\section{Homogeneous g.o. spaces}

2.1. Homogeneous Riemannian spaces. A Riemannian manifold $\left(Q, d s^{2}\right)$ is homogeneous if it admits a transitive connected Lie group of isometries. Then $Q$ can be seen as a coset space $G / H$ with a $G$-invariant metric, where $H$ is the isotropy group at some point $o \in Q$. 
Since $H$ is compact, there exist an $\operatorname{Ad}_{H}$-invariant subspace $\mathfrak{v} \subset \mathfrak{g}([\mathfrak{h}, \mathfrak{v}] \subset \mathfrak{v})$ such that $\mathfrak{g}=\mathfrak{h} \oplus \mathfrak{v}$ (a reductive decomposition of $\mathfrak{g}$ ). Here $\mathfrak{g}$ and $\mathfrak{h}$ are Lie algebras of $G$ and $H$ and $\operatorname{Ad}_{H}$ is the restriction of the adjoint representation $\operatorname{Ad}_{G}$ to $H$.

We can identify $\mathfrak{v}$ with $T_{o} Q$ by taking the value of the corresponding Killing vector field at $o$. In this way, the isotropy representation of $H$ at $T_{o} Q$ is identified with the restriction of the adjoint representation of $H$ to $\mathfrak{v}$. The restriction of a $G$-invariant metrics $d s^{2}$ to $T_{o} Q \cong \mathfrak{v}$ defines $\operatorname{Ad}_{H}$-invariant scalar product $(\cdot, \cdot)$ on $\mathfrak{v}$. Conversely, for each $\operatorname{Ad}_{H}$-invariant scalar product $(\cdot, \cdot)$ on $\mathfrak{v}$, there exist an unique $G$-invariant Riemannian metric $d s^{2}$ on $Q=G / H$ such that its restriction to $T_{o} Q \cong \mathfrak{v}$ is given by $(\cdot, \cdot)$ (e.g., see [7]).

A $G$-invariant metric $d s_{0}^{2}$ is called normal if $(\cdot, \cdot)$ is the restriction of an $\operatorname{Ad}_{G}$-invariant non-degenerate symmetric bilinear form $q$ on $\mathfrak{g}$. If $G$ is semi-simple and $q=-B$ ( $B$ is the Killing form), the metric $d s_{0}^{2}$ is called standard.

A metric $d s^{2}$ is naturally reductive if

$$
\left([X, Y]_{\mathfrak{v}}, Z\right)+\left(Y,[X, Z]_{\mathfrak{v}}\right)=0, \quad X, Y, Z \in \mathfrak{v} .
$$

Clearly, a normal metric is naturally reductive. A notion of natural reductivity depends on the choice of the group $G$ and the choice of $\mathfrak{v}$. A result of Konstant (e.g., see 7], page 196) stated that $\left(Q=G / H, d s^{2}\right)$ is naturally reductive if and only if there exist a subgroup $\tilde{G} \subset G$ with the Lie algebra $\tilde{\mathfrak{g}}=\mathfrak{v}+[\mathfrak{v}, \mathfrak{v}]$, such that $d s^{2}$ is a normal metric on $Q=\tilde{G} /(\tilde{G} \cap H)$.

2.2. Riemannian g.o. spaces The condition (5) is equivalent to the following geometrical property: the curve $\gamma(t)=\exp (t X) \cdot o$ is a geodesic for all $X \in \mathfrak{v}$. Thus, for a naturally reductive homogeneous space every geodesic is homogeneous. By generalizing the property above, Kowalski and Vanhecke [20 introduced a notion of g.o. spaces. A Riemannian homogeneous space $\left(Q=G / H, d s^{2}\right)$ is a g.o. space, if every geodesic is an orbit of an one-parameter subgroup $\exp (t X), X \in \mathfrak{g}$.

The following algebraic condition on an orbit to be a geodesic is well known [20]:

Lemma 1 (Geodesic lemma): Let $X \in \mathfrak{v}, F \in \mathfrak{h}$. The orbit $\gamma(t)=\exp (t(X+F)) \cdot o$ through the point $o=[H] \in G / H$ is a geodesic of $\left(Q=G / H, d s^{2}\right)$ if and only if

$$
\left([X+F, Y]_{\mathfrak{v}}, X\right)=0, \quad \text { for all } \quad Y \in \mathfrak{v} .
$$

Therefore, a Riemannian homogeneous space $\left(Q=G / H, d s^{2}\right)$ is a g.o. space if and only if for any vector $X \in \mathfrak{v}$ there exist $F=F(X) \in \mathfrak{h}$ that satisfies (6).

Examples of such spaces are symmetric spaces, weakly symmetric spaces (for any two points $p, q \in Q$, there exist an isometry which interchanges $p$ and $q$ [32, [5, 6, 37, 38, 40]) and naturally reductive spaces.

2.3. $G$-invariant functions and a characterization of g.o. metrics. From now on $G$ is a compact connected Lie group $G$. We fix an $\operatorname{Ad}_{G}$-invariant scalar product $\langle\cdot, \cdot\rangle$ on $\mathfrak{g}$. Then $\mathfrak{g}=\mathfrak{h} \oplus \mathfrak{v}, \mathfrak{v}=\mathfrak{h}^{\perp}$ is a reductive decomposition of $\mathfrak{g}$. An $\operatorname{Ad}_{H}$-invariant scalar product on $\mathfrak{v} \cong T_{o} Q$ can be represented as

$$
(\cdot, \cdot)=\langle I(\cdot), \cdot\rangle,
$$

where $I: \mathfrak{v} \rightarrow \mathfrak{v}$ is a positive definite, $\operatorname{Ad}_{H}$-invariant operator. For a given operator $I$, we denote the associated $G$-invariant metric by $d s_{I}^{2}$. In particular, for $I$ equals to the identity, we have a normal metric $d s_{0}^{2}$.

By using $d s_{0}^{2}$ we identify tangent and cotangent bundles $T Q \cong T^{*} Q$. Within this identification, the $G$-invariant functions are in one-to-one correspondence with $\operatorname{Ad}_{H}$-invariant polynomials $\mathbb{R}[\mathfrak{v}]^{H}$ on $\mathfrak{v}$. The Hamiltonian of the metric $d s_{I}^{2}$ is given by $h_{A}(x)=\frac{1}{2}\langle A x, x\rangle$ where $A=I^{-1}$, while the Hamiltonian of the normal metric $d s_{0}^{2}$ is simply $h_{0}(x)=\frac{1}{2}\langle x, x\rangle$. 
Further, the canonical Poisson bracket on $T^{*} Q$ corresponds to the restriction of the Lie-Poisson bracket to $\mathbb{R}[\mathfrak{v}]^{H}$ (see [35]):

$$
\{f, g\}_{\mathfrak{v}}(x)=-\langle x,[\nabla f(x), \nabla g(x)]\rangle, \quad f, g: \mathfrak{v} \rightarrow \mathbb{R} .
$$

Let $\mathfrak{g}_{x}$ and $\mathfrak{h}_{x}$ be the isotropy algebras of $x$ in $\mathfrak{g}$ and $\mathfrak{h}$ and let $\mathfrak{j}_{x} \subset \mathfrak{v}$ be the orthogonal complement to the orbit $\operatorname{Ad}_{H}(x) \subset \mathfrak{v}: \mathfrak{j}_{x}=\{\eta \in \mathfrak{v} \mid\langle\eta,[x, \mathfrak{h}]\rangle=0\}$. For a generic point $x \in \mathfrak{v}$, it is spanned by gradients of polynomials in $\mathbb{R}[\mathfrak{v}]^{H}$. The maximal number of functionally independent polynomials is equal to

$$
\operatorname{ddim} \mathcal{F}_{G}=\operatorname{ddim} \mathbb{R}[\mathfrak{v}]^{H}=\operatorname{dim} \mathfrak{j}_{x}=\operatorname{dim} \mathfrak{v}-\operatorname{dim} \mathfrak{h}+\operatorname{dim} \mathfrak{h}_{x},
$$

for a generic $x \in \mathfrak{v}$.

An $\operatorname{Ad}_{H}$-invariant polynomial $f(x)$ is central (Casimir) within $\mathbb{R}[\mathfrak{v}]^{H}$ if it commutes with all invariant polynomials, that is $\nabla f(x) \in \operatorname{ker} \Lambda_{x}$, where

$$
\Lambda_{x}\left(\eta_{1}, \eta_{2}\right)=-\left\langle x,\left[\eta_{1}, \eta_{2}\right]\right\rangle, \quad \eta_{1}, \eta_{2} \in \mathfrak{j}_{x} .
$$

The kernel of $\Lambda_{x}$ is $\operatorname{ker} \Lambda_{x}=\operatorname{pr}_{\mathfrak{v}} \mathfrak{g}_{x} \subset \mathfrak{j}_{x}$. Thus, the maximal number of functionally independent central polynomials is dind $\mathbb{R}[\mathfrak{v}]^{H}=\operatorname{dim} \operatorname{ker} \Lambda_{x}=\operatorname{dim} \mathfrak{g}_{x}-\operatorname{dim} \mathfrak{h}_{x}$, for a generic $x \in \mathfrak{v}\left[9\right.$, 10. Moreover, since $\left[\mathfrak{g}_{x}, \mathfrak{g}_{x}\right] \subset \mathfrak{h}_{x}$ (see Mykytyuk [25]) we have also:

$$
\operatorname{dind} \mathcal{F}_{G}=\operatorname{dind} \mathbb{R}[\mathfrak{v}]^{H}=\operatorname{dim} \mathfrak{g}_{x}-\operatorname{dim} \mathfrak{h}_{x}=\operatorname{rank} \mathfrak{g}-\operatorname{rank} \mathfrak{h}_{x},
$$

for a generic $x \in \mathfrak{v}$.

Let $f_{1}, \ldots, f_{r}$ be a base of homogeneous $\operatorname{Ad}_{G}$-invariant polynomials on $\mathfrak{g}(r=\operatorname{rank} \mathfrak{g})$. They are central functions with respect to the Lie-Poisson brackets on $\mathfrak{g}$, while their restrictions $p_{i}=\left.f_{i}\right|_{\mathfrak{v}}$ to $\mathfrak{v}$ are central functions in $\mathbb{R}[\mathfrak{v}]^{H}$. Among $p_{1}, \ldots, p_{r}$ there are dind $\mathbb{R}[\mathfrak{v}]^{H}$ functionally independent polynomials. In particular, a normal metric Hamiltonian $h_{0}(x)=$ $\frac{1}{2}\langle x, x\rangle$ is a central function.

Lemma 2: A Riemannian homogeneous space $\left(Q=G / H, d s_{I}^{2}\right)$ is a g.o. space if and only if the Hamiltonian $h_{A}(x)=\frac{1}{2}\langle A x, x\rangle$ is a central function in $\mathbb{R}[\mathfrak{v}]^{H}$.

Proof. The Hamiltonian $h_{A}(x)=\frac{1}{2}\langle A x, x\rangle$ is $\operatorname{Ad}_{H}$-invariant implying

$$
\operatorname{ad}_{\mathfrak{h}}(x) \cdot h_{A}(x)=0 \quad \Longleftrightarrow \quad[x, A x]_{\mathfrak{h}}=0 .
$$

According to Lemma $1,\left(Q=G / H, d s_{I}^{2}\right)$ is a g.o. space if and only if for every $X \in \mathfrak{v}$ there exist $F(X) \in \mathfrak{h}$ satisfying

$$
\left\langle[X+F(X), Y]_{\mathfrak{v}}, I X\right\rangle=0, \quad \text { for all } \quad Y \in \mathfrak{v} .
$$

Denote $x=I X, a=a(x)=F(A x)$. Then we can rewrite (11) as

$$
[a(x)+A x, x]_{\mathfrak{v}}=0 .
$$

Since $[a, x] \in \mathfrak{v}$, the relation (10) implies equivalence of (12) and

$$
[a(x)+A x, x]=0 \quad \Longleftrightarrow \quad A x=\nabla h_{A}(x) \in \operatorname{pr}_{\mathfrak{v}} \mathfrak{g}_{x}=\operatorname{ker} \Lambda_{x} .
$$

Therefore $\left(Q=G / H, d s_{I}^{2}\right)$ is a g.o. space if and only if $h_{A}(x)$ is a central function in $\mathbb{R}[\mathfrak{v}]^{H}$.

Remark 1: The geodesic flows of a g.o. metric $d s_{I}^{2}$ and of a normal metric $d s_{0}^{2}$ share the same invariant isotropic toric foliation defined by integrals (11) (a generic torus has a dimension dind $\left.\mathcal{F}=\operatorname{dind} \mathcal{F}_{G}=\operatorname{dind} \Phi^{*}\left(\mathbb{R}\left[\mathfrak{g}^{*}\right]\right)\right)$. However, the tori can be resonant and the closures of geodesic lines might be different. For example, while the geodesic lines of the standard metric on a sphere are great circles, we see that generic geodesic lines on the distance spheres $S^{4 n+3}=S p(n+1) / S p(n)$ and $S^{2 n+1}=S U(n+1) / S U(n)$, described in Examples 5 and 6 . filled up regions that are projections of two-dimensional invariant isotropic tori. 
2.4. Examples. Here we list some known examples of g.o. spaces with compact groups of isometries and with non-normal metrics.

Aleksieevsky and Arvanitoyeorgos proved that among all flag manifolds $Q=G / H$ of simple Lie groups $G$ only the manifolds

$$
S O(2 n+1) / U(n), \quad S p(n) / U(1) \times S p(n-1)
$$

admit invariant metrics with homogeneous geodesics, not homothetic to the standard metric. These manifolds have an one-parameter family of metrics $d s_{\lambda}^{2}, \lambda>0$ with homogeneous geodesics and are all weakly symmetric, see 40 . The metric $d s_{1}^{2}$ is the standard one. It has the full connected isometry group $S O(2 n+2)$ (respectively $S U(2 n-1)$ ) and is the standard metric of the symmetric space $S O(2 n+2) / U(n+1)$ (respectively the complex projective space $\left.\mathbb{C P}^{2 n-1}=S U(2 n-1) / U(2 n-2)\right)$. All the other metrics have the full connected isometry group $S O(2 n+1)$ (respectively $S p(n)$ ) and the corresponding spaces are not naturally reductive. This two classes of examples exhaust all simply connected compact irreducible Riemannian non-normal g.o. manifolds of positive Euler characteristic (see Aleksieevsky and Nikonorov [2]).

For $l=2, S p(2) / U(1) \cdot S p(1) \cong S O(5) / U(2)$ is the 6 -dimensional not naturally reductive g.o. space of Kowalski and Vanhecke, who classified all g.o. spaces in dimension $\leq 6[20]$.

Dušek, Kowalski and Nikčević constructed a family of invariant metrics $d s_{p, q}^{2}$ on a 7 dimensional homogeneous space $Q=(S O(5) \times S O(2)) / U(2)$, such that $\left(Q, d s_{p, q}^{2}\right)$ are g.o. spaces which are not naturally reductive 13. Besides, the group $S O(5)$ acts as a transitive group of isometries, but the homogeneous spaces $\left(Q=S O(5) / S U(2), d s_{p, q}^{2}\right)$ are not g.o. spaces. Note that $(S O(5) \times S O(2)) / U(2)$ is also a weakly symmetric space (see the classification of weakly symmetric spaces given by Yakimova [38]).

Finally, Tamaru classified the Riemannian, non-standard g.o. spaces, which are fibered over the irreducible symmetric spaces [33, 34] (see Table 1 below). In particular, the flag manifolds (13) belong to the considered class of homogeneous spaces. They are associated to the triples $(S O(2 n+1), S O(2 n), U(n))$ and $(S p(n), S p(1) \times S p(n-1), U(1) \times S p(n-1))$, respectively. Note that the spaces listed in Table 1 can be naturally reductive with respect to a suitably larger symmetry group (e.g., see Kowalski and Nikčević 21 for the space $\left.S U(3) / S U(2) \cong S^{5}\right)$.

\section{Geodesic flows on fiber bundles}

3.1. Integrable pairs and complexity of homogeneous spaces. Recall that for a complete set of integrals (11), the Mishchenko-Fomenko conjecture reduces to a construction of a commutative subset $\mathcal{B} \subset \mathcal{F}_{G}$ that satisfies condition (3). If such a set exists, we call $(G, H)$ an integrable pair. If $G / H$ is a weakly symmetric space, in particular if $G / H$ is a symmetric space, the algebra $\mathcal{F}_{G}$ is already commutative [37. In this case the pair $(G, H)$ is obviously integrable and we need only Noether's integrals to integrate the geodesic flow (see [11, 25]). Further examples can be found in [35, 27, 8, 10, 26, 12, 18].

There is a well known notion of homogeneous spaces complexity of complex reductive Lie groups [30. In the case of a homogeneous space $G / H$ of a compact Lie group $G$, the complexity of $G^{\mathbb{C}} / H^{\mathbb{C}}$ corresponds to the number of independent polynomials, apart from the central polynomials, which we need to form a complete commutative subset $\mathcal{B} \subset \mathbb{R}[\mathfrak{v}]^{H}$ (see Mykytyuk [28]). We refer to

$$
c(G, H)=\frac{1}{2}\left(\operatorname{ddim} \mathbb{R}[\mathfrak{v}]^{H}-\operatorname{dind} \mathbb{R}[\mathfrak{v}]^{H}\right),
$$

as a complexity of the homogeneous space $G / H$ or the pair $(G, H)$. 
Symmetric and weakly symmetric spaces have the complexity $c=0$ [37. The pairs $(G, H)$ with the complexity $c=1$ are also integrable: we can take an arbitrary non central polynomial and the central polynomials to form a required commutative set $\mathcal{B}$ (see Mykytyuk and Stepin 27]). The classification of the complexity 1 homogeneous spaces of reductive algebraic groups is given by Arzhantsev and Chuvashova [4] (see also [30, 27]).

3.2. Triples of Lie groups. Suppose $G$ is a connected compact semi-simple Lie group. Let $H \subset K \subset G$ be a chain of subgroups and $\mathfrak{h} \subset \mathfrak{k} \subset \mathfrak{g}$ be the appropriate chain of subalgebras.

Let $\langle\cdot, \cdot\rangle$ to be the negative of the Killing form on $\mathfrak{g}$. Taking the suitable orthogonal complements, we obtain decompositions

$$
\mathfrak{g}=\mathfrak{h} \oplus \mathfrak{v}=\mathfrak{h} \oplus \mathfrak{l} \oplus \mathfrak{m}, \quad \mathfrak{k}=\mathfrak{h} \oplus \mathfrak{l} .
$$

Following [33, 34, consider a deformation $d s_{\lambda}^{2}$ of the standard metric on $Q=G / H$ in the direction of the fiber $K / H$ of the bundle (44), defined as a scalar product on $\mathfrak{v}=\mathfrak{l} \oplus \mathfrak{m} \cong T_{o} Q$ :

$$
(\cdot, \cdot)_{\lambda}=\lambda\langle\cdot, \cdot\rangle_{\mathfrak{l} \times \mathfrak{l}}+\langle\cdot, \cdot\rangle_{\mathfrak{m} \times \mathfrak{m}}, \quad \lambda>0 .
$$

The Hamiltonian of the metric $d s_{\lambda}^{2}$ is a deformation of the Hamiltonian of the standard metric $d s_{0}^{2}$ :

$$
h_{\lambda}(x)=h_{0}(x)+\left(\lambda^{-1}-1\right) \delta(x), \quad h_{0}(x)=\frac{1}{2}\left\langle x_{\mathfrak{m}}, x_{\mathfrak{m}}\right\rangle+\frac{1}{2}\left\langle x_{\mathfrak{l}}, x_{\mathfrak{l}}\right\rangle, \quad \delta(x)=\frac{1}{2}\left\langle x_{\mathfrak{l}}, x_{\mathfrak{l}}\right\rangle,
$$

where $x=x_{\mathfrak{l}}+x_{\mathfrak{m}}, x_{\mathfrak{l}} \in \mathfrak{l}, x_{\mathfrak{m}} \in \mathfrak{m}$.

A natural problem is whether the geodesic flow of $d s_{\lambda}^{2}$ is integrable. The positive answer for a class of spaces is given in [10. Here we give the following simple criterium.

Theorem 3: Let $(G, K, H)$ be a triple of connected Lie groups such that $[\mathfrak{l}, \mathfrak{h}]=0$ and $[\mathfrak{l}, \mathfrak{l}] \subset \mathfrak{l}$. Then

(i) the geodesic flow of the metric $d s_{\lambda}^{2}$ is noncommutative integrable;

(ii) if $\mathfrak{l}$ is commutative ( $\mathfrak{l}$ is a subset of the center of $\mathfrak{k}$ ) and $(G, K)$ is an integrable pair then $(G, H)$ is also an integrable pair.

Proof. (i) Let $L$ be the connected subgroup of $K$ with the Lie algebra l. Then $H$ and $L$ are normal subgroups of $K$. Locally, $K$ is isomorphic to the product $H \times L$. The right translations on $\left(Q=G / H, d s_{\lambda}^{2}\right)$ with elements of $L$ are well defined isometries. Note that the actions of $G$ and $L$ commute and that (44) is a $L$-principal bundle.

Let $\Psi: T^{*} Q \rightarrow \mathfrak{l}^{*}$ be the momentum mapping of the $L$-action lifted to the cotangent bundle and let $\mathcal{F}_{G \times L}$ be the algebra of $G \times L$-invariant analytic, polynomial in momenta functions on $T^{*} Q$. By the general construction [9], the collection of functions

$$
\mathcal{F}_{G \times L}+\Psi^{*}\left(\mathbb{R}\left[\mathfrak{l}^{*}\right]\right)+\Phi^{*}\left(\mathbb{R}\left[\mathfrak{g}^{*}\right]\right)
$$

is a complete set on $T^{*} Q$. It remains to observe that these functions commute both with $h_{0}(x)$ and $\delta(x)$.

(ii) Within the identification $\mathcal{F}_{G} \cong \mathbb{R}[\mathfrak{v}]^{H}$, the algebra $\Psi^{*}\left(\mathbb{R}\left[\mathfrak{l}^{*}\right]\right)$ corresponds to the algebra $\mathcal{L}$ generated by linear functions on $\mathfrak{l}$, extended to $\mathfrak{v}$ via decomposition (14).

Assume $\mathcal{B}_{M}$ is a complete commutative set in $\mathbb{R}[\mathfrak{m}]^{K}$. Let $\mathcal{B}=\mathcal{B}_{M}+\mathcal{L}$, where the polynomials in $\mathcal{B}_{M}$ are extended to $\mathfrak{v}$ via decomposition (14). It is clear that $\mathcal{B}$ is a commutative subset of $\mathbb{R}[\mathfrak{v}]^{H}$. Indeed, from $[\mathfrak{l}, \mathfrak{k}]=0$ we get $\{\mathcal{L}, \mathcal{L}\}_{\mathfrak{v}}=0$ and $\left\{\mathcal{B}_{M}, \mathcal{L}\right\}_{\mathfrak{v}}=0$ follows from $\operatorname{Ad}_{K}$-invariancy of polynomials in $\mathcal{B}_{M}$. Furthermore, since

$$
\operatorname{dim} \mathfrak{g}_{x} \leq \operatorname{dim} g_{x_{\mathfrak{m}}}, \quad \text { for a generic } \quad x \in \mathfrak{v}, x_{\mathfrak{m}} \in \mathfrak{m},
$$


we get

$$
\begin{aligned}
\operatorname{ddim} \mathcal{B} & =\operatorname{ddim} \mathcal{B}_{M}+\operatorname{dim} \mathfrak{l}=\frac{1}{2}\left(\operatorname{ddim} \mathbb{R}[\mathfrak{m}]^{K}+\operatorname{dind} \mathbb{R}[\mathfrak{m}]^{K}\right)+\operatorname{dim} \mathfrak{l} \\
& =\frac{1}{2}\left(\operatorname{dim} \mathfrak{m}-\operatorname{dim} \mathfrak{k}+\operatorname{dim} \mathfrak{g}_{x_{\mathfrak{m}}}\right)+\operatorname{dim} \mathfrak{l}=\frac{1}{2}\left(\operatorname{dim} \mathfrak{v}-\operatorname{dim} \mathfrak{h}+\operatorname{dim} \mathfrak{g}_{x_{\mathfrak{m}}}\right) \\
& \geq \frac{1}{2}\left(\operatorname{ddim} \mathbb{R}[\mathfrak{v}]^{H}+\operatorname{dind} \mathbb{R}[\mathfrak{v}]^{H}\right) .
\end{aligned}
$$

On the other hand, for a commutative set $\mathcal{B}$ we always have the inequality ddim $\mathcal{B} \leq$ $\frac{1}{2}\left(\operatorname{ddim} \mathbb{R}[\mathfrak{v}]^{H}+\operatorname{dind} \mathbb{R}[\mathfrak{v}]^{H}\right)$. Therefore, (17) is an equality and $\mathcal{B}$ is a complete set.

Example 1: The homogeneous spaces considered in Theorem 3 are studied from a point of view of a construction of invariant Einstein metrics [16 (see also 7]). Jensen classified Einstein metrics of the form $d s_{\lambda}^{2}$ on homogeneous spaces $Q=G / H$ fibered over irreducible symmetric spaces $M=G / K$. When $\mathfrak{l}$ is nonabelian, then there are two Einstein metrics, neither of them equals to the standard one. When $\mathfrak{l}$ is commutative, there is only one Einstein metric, which equals to the standard one when $\operatorname{dim} L=1$ and $\operatorname{dim} M=2$ [16]. For example, the triple $(S O(n), S O(r) \times S O(n-r), S O(n-r))$ provides two Einstein metrics on a Stiefel variety $V_{n, r}=S O(n) / S O(n-r)$. The geometry and integrability of natural mechanical systems on Stiefel varieties $V_{n, r}$ endowed with metrics $d s_{\lambda}^{2}$ and with quadratic potentials is studied in [14].

Example 2: A metric $d s_{\lambda}^{2}$ can be always additionally perturb by taking an arbitrary integrable Euler equation on $\mathfrak{l} \cong \mathfrak{l}^{*}$

$$
\dot{x}_{\mathfrak{l}}=\left[x_{\mathfrak{l}}, A x_{\mathfrak{l}}\right], \quad A: \mathfrak{l} \rightarrow \mathfrak{l}
$$

with a complete set of integrals $\mathcal{S}$. Namely, define $h_{\lambda, A}=h_{\lambda}(x)+\frac{1}{2}\left\langle x_{\mathfrak{l}}, A x_{\mathfrak{l}}\right\rangle$. We can find $\lambda$ such that $h_{\lambda, A}$ is positive definite. It is a Hamiltonian of a suitable $G$-invariant metric $d s_{\lambda, A}^{2}$. From the completeness of sets $\mathcal{S}$ on $\mathfrak{l}$ and (16) on $T^{*} Q$, we get that $\mathcal{F}_{G \times L}+\mathcal{S}$ is a complete subset of $\mathcal{F}_{G} \cong \mathbb{R}[\mathfrak{v}]^{H}$ :

$$
\operatorname{ddim}\left(\mathcal{F}_{G \times L}+\mathcal{S}\right)+\operatorname{dind}\left(\mathcal{F}_{G \times L}+\mathcal{S}\right)=\operatorname{ddim} \mathbb{R}[\mathfrak{v}]^{H}+\operatorname{dind} \mathbb{R}[\mathfrak{v}]^{H} .
$$

Thus, the geodesic flow of $d s_{\lambda, A}^{2}$ is completely integrable. Note that Einstein metrics on Stiefel manifolds $V_{n, r}$ obtained in [3] are of the form $d s_{\lambda, A}^{2}[14$.

Example 3: As an application of Theorem 3, item (ii), let us consider integrable pairs $\left(S p(n), U\left(k_{1}\right) \times \cdots \times U\left(k_{r}\right)\right)\left(k_{i} \leq[(n+1) / 2]\right.$, see [10] $)$. Then we get that $\left(S p(n), S U\left(k_{1}\right) \times\right.$ $\left.\cdots \times S U\left(k_{r}\right)\right)$ are integrable pairs as well.

\section{G.O. spaces fibered over symmetric spaces}

4.1. Consider a triple of Lie groups $(G, K, H)$. According to Lemma 2. $\left(G / H, d s_{\lambda}^{2}\right)$ is a g.o. space if and only if $\delta(x)$ is a central function in $\mathbb{R}[\mathfrak{v}]^{H}$, that is $\nabla \delta(x)=x_{\mathfrak{l}} \in \operatorname{pr}_{\mathfrak{v}} \mathfrak{g}_{x}$. In such a way we obtain the following condition (see Gordon [15]): $\left(G / H, d s_{\lambda}^{2}\right)$ is a g.o. space if and only if for every $x \in \mathfrak{v}$, there exist $a=a(x) \in \mathfrak{h}$, that satisfies

$$
\left[a+x_{\mathfrak{l}}, x_{\mathfrak{l}}+x_{\mathfrak{k}}\right]=0 \quad \Longleftrightarrow \quad\left[a, x_{\mathfrak{l}}\right]=0, \quad\left[a+x_{\mathfrak{l}}, x_{\mathfrak{m}}\right]=0 .
$$

In particular, if $\left(G / H, d s_{\lambda}^{2}\right)$ is a g.o. space for a single $\lambda \neq 1$, then it is a g.o. space for all $\lambda>0$. Triples $(G, K, H)$ where $(G, K)$ is a compact effective irreducible symmetric pair and $\left(G / H, d s_{\lambda}^{2}\right)$ is a g.o. space are classified by Tamaru [34]. 


\begin{tabular}{|c|c|c|c|c|}
\hline & $\overline{\mathfrak{g}}$ & $\mathfrak{k}$ & $\mathfrak{h}$ & \\
\hline 1 & $s o(2 n+1)$ & $s o(2 n)$ & $u(n)$ & $n \geq 2$ \\
\hline 2 & $s o(4 n+1)$ & $s o(4 n)$ & $s u(2 n)$ & $n \geq 1$ \\
\hline 3 & $s o(8)$ & so(7) & $g_{2}$ & \\
\hline 4 & $s o(9)$ & so(8) & $\operatorname{spin}(7)$ & \\
\hline 5 & $s u(n+1)$ & $u(n)$ & $s u(n)$ & $n \geq 2$ \\
\hline 6 & $s u(2 n+1)$ & $u(2 n)$ & $u(1) \oplus \operatorname{sp}(n)$ & $n \geq 2$ \\
\hline 7 & $s u(2 n+1)$ & $u(2 n)$ & $s p(n)$ & $n \geq 2$ \\
\hline 8 & $s p(n+1)$ & $s p(1) \oplus \operatorname{sp}(n)$ & $u(1) \oplus \operatorname{sp}(n)$ & $n \geq 1$ \\
\hline 9 & $s p(n+1)$ & $s p(1) \oplus s p(n)$ & $s p(n)$ & $n \geq 1$ \\
\hline 10 & $s u(2 r+n)$ & $s u(r) \oplus s u(r+n) \oplus \mathbb{R}$ & $s u(r) \oplus s u(r+n)$ & $r \geq 2, n \geq 1$ \\
\hline 11 & $s o(4 r+2)$ & $u(2 r+1)$ & $s u(2 r+1)$ & $r \geq 2$ \\
\hline 12 & $e_{6}$ & $s o(10) \oplus \mathbb{R}$ & $s o(10)$ & \\
\hline 13 & so(9) & $s o(7) \oplus s o(2)$ & $g_{2} \oplus s o(2)$ & \\
\hline 14 & $s o(10)$ & $s o(8) \oplus s o(2)$ & $\operatorname{spin}(7) \oplus \operatorname{so}(2)$ & \\
\hline 15 & so(11) & $s o(8) \oplus s o(3)$ & $\operatorname{spin}(7) \oplus \operatorname{so}(3)$ & \\
\hline
\end{tabular}

Table 1. Tamaru's classification 34]

Theorem 4: All pairs $(G, H)$ associated to the triples of Lie algebras $(\mathfrak{g}, \mathfrak{k}, \mathfrak{h})$ listed in Table 1 are integrable. The geodesic flows on g.o. spaces $\left(G / H, d s_{\lambda}^{2}\right)$ are Liouville integrable by means of analytic integrals, polynomial in momenta.

Proof. Integrability of the pairs $(G, H)$ in the cases $1,2,9,5,8$ is described, respectively, in Examples 4, 5, 6] and 7. while in the cases 10, 11, 12 it follows from Theorem 3, item (ii) and from the fact that symmetric pairs are integrable. Further, we can simply use the known classifications of complexity 0 and 1 homogeneous spaces given in [25, 38, 30, 27, 4, to conclude that the others homogeneous spaces in Tamaru's classification have the complexity equal 0 or 1 as well. Thus, all pairs $(G, H)$ associated to the triples of Lie algebras $(\mathfrak{g}, \mathfrak{k}, \mathfrak{h})$ listed in Table 1 are integrable.

Remark 2: Up to the covering, which preserves the integrability of geodesic flows, the g.o. spaces of compact simple Lie groups obtained in [20, 1, 33] are also included in Table 1 (for example, the weakly symmetric spaces $\operatorname{Spin}(8) / G_{2}$ and $\operatorname{Spin}(9) / \operatorname{Spin}(7)$ [40, 33. correspond to the cases 3 and 4$)$. On the other hand, since $(S O(5) \times S O(2)) / U(2)$ is a weakly symmetric space [38, the g.o. spaces $\left((S O(5) \times S O(2)) / U(2), d s_{p, q}^{2}\right)$ constructed in 13] have Liouville integrable geodesic flows as well.

4.2. Under the assumption of Theorem [3, item (ii), if $\operatorname{dim} \mathfrak{l}=1$ and the complexity of $G / K$ is equal to 0 , we get that the complexity of the homogeneous space $G / H$ is either equal to 0 (a linear function on $\mathfrak{l}$ is a central function of $\mathbb{R}[\mathfrak{v}]^{H}$ ) or 1 (a linear function on $\mathfrak{l}$ is not a central function), see also Remark 6 in [27.

For example, the flag manifolds (13), as weakly symmetric spaces have the complexity $c=0$. Starting from $S O(2 n+1) / U(n)$ and $S p(n) / U(1) \times S p(n-1)$ we obtain the complexity one homogeneous spaces $S O(2 n+1) / S U(n)$ and $S p(n+1) / S p(n)$. The spaces $S p(n) / U(1) \times$ $S p(n-1)$ and $S p(n+1) / S p(n)$ will be treated in Examples 7 and 5 below. In the next example we derive the complexity of homogeneous spaces $S O(2 n+1) / U(n)$ and $S O(2 n+$ 1) $/ S U(n)$. 
Example 4: Consider a triple of Lie groups $(S O(2 n+1), U(n), S U(n))$. The inclusion $u(n) \subset$ $s o(2 n+1)$ is the standard one, given by the mapping

$$
P+i Q \in u(n) \longmapsto\left(\begin{array}{ccc}
P & Q & 0 \\
-Q & P & 0 \\
0 & 0 & 0
\end{array}\right) \in s o(2 n+1) .
$$

Let us take $x_{\mathfrak{m}}=x_{1}+x_{2}$, where

$$
\begin{aligned}
& x_{1}=\alpha_{1}\left(E_{1} \wedge E_{2}-E_{2 m+1} \wedge E_{2 m+2}\right)+\cdots+\alpha_{m}\left(E_{2 m-1} \wedge E_{2 m}-E_{4 m-1} \wedge E_{4 m}\right), \\
& x_{2}=\beta_{1} E_{1} \wedge E_{4 m+1}+\cdots+\beta_{4 m} E_{1} \wedge E_{4 m+1}, \quad m=[n / 2] .
\end{aligned}
$$

Straightforward computations yield $u(n)_{x_{1}} \cong s u(2)^{m} \oplus u(1)^{n-2 m}, u(n)_{x_{2}} \cong u(n-1)$ and

$$
u(n)_{x_{\mathrm{m}}}=u(n)_{x_{1}} \cap u(n)_{x_{2}}=\{0\},
$$

for a generic values of the parameters $\left(\alpha_{1}, \ldots, \alpha_{m}\right)$ and $\left(\beta_{1}, \ldots, \beta_{4 m}\right)$. Thus, $\operatorname{dim} \mathfrak{u}(n)_{x_{\mathfrak{m}}}=0$, for a generic $x \in \mathfrak{m}$.

Further, $\operatorname{dim} \mathfrak{m}-\operatorname{dim} u(n)=\operatorname{dim} s o(2 n+1)-2 \operatorname{dim} u(n)=n=\operatorname{rank} s o(2 n+1)$. Therefore, $\operatorname{ddim} \mathbb{R}[\mathfrak{m}]^{U(n)}=\operatorname{dind} \mathbb{R}[\mathfrak{m}]^{U(n)}=n$ (see (18) and (9) $)$ and $c(S O(2 n+1), U(n))=0$. A complete set of independent commuting $\operatorname{Ad}_{U(n)}$-invariant polynomials is simply

$$
p_{1}=\operatorname{tr}\left(x^{2}\right), p_{2}=\operatorname{tr}\left(x^{4}\right), \ldots, p_{n}=\operatorname{tr}\left(x^{2 n}\right) .
$$

Next, according to item (ii) of Theorem 3 a complete commutative set on $\mathfrak{v}$ has dind $\mathbb{R}[\mathfrak{m}]^{U(n)}+$ $1=\operatorname{rank} s o(2 n+1)+1$ independent functions. Thus, $c(S O(2 n+1), S U(n))=1$.

Example 5: $(G, K, H)=(S p(n+1), S p(1) \times S p(n), S p(n)), G / H=S^{4 n+3}$. We have $\mathfrak{v}=$

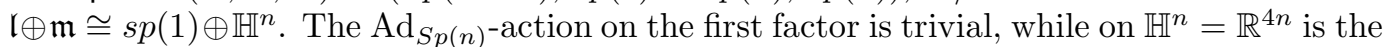
standard one: the orbit of $e \in \mathbb{H}^{n}$ is a sphere $S^{4 n-1} \cong S p(n) / S p(n-1)$ (see [39]). Therefore, the algebra $\mathbb{R}[\mathfrak{v}]^{S p(n)}$ is generated by the linear functions on $\operatorname{sp}(1)$ and a quadratic invariant on $\mathbb{H}^{n}$, while the center of $\mathbb{R}[\mathfrak{v}]^{S p(n)}$ is generated by quadratic invariants on $s p(1)$ and $\mathbb{H}^{n}$ (for example $\delta(x)$ and $h_{0}(x)$ ). Thus

$$
\operatorname{ddim} \mathbb{R}[\mathfrak{v}]^{S p(n)}=4, \quad \operatorname{dind} \mathbb{R}[\mathfrak{v}]^{S p(n)}=2 .
$$

For a complete commutative set $\mathcal{B}$ we can take an arbitrary linear function $f$ on $s p(1)$, together with $\delta(x)$ and $h_{0}(x)$. In particular we get the complexity $c(S p(n+1), S p(n))=1$.

The sphere $\left(S^{4 n+3}=S p(n+1) / S p(n), d s_{\lambda}^{2}\right)$ has $S p(n+1) \times S p(1)$ as a full group of isometries (see the proof of Theorem [3, item (i)) and

$$
S^{4 n+3}=S p(n+1) \times S p(1) / S p(n) \times S p(1)
$$

is a weakly symmetric space, a distance sphere in a quaternionic projective space $\mathbb{H}_{\mathbb{P}^{n+1}}=$ $S p(n+2) / S p(n+1) \times S p(1)($ see $[39,5)$. From the analysis above, we see that the commutative algebra of $S p(n+1) \times S p(n)$-invariant polynomials on $T^{*} S^{4 n+3}$ is generated by $\delta(x)$ and $h_{0}(x)$.

Example 6: $(G, K, H)=(S U(n+1), U(n), S U(n)), G / H=S^{2 n+1} . \quad$ Now, $\mathfrak{v}=\mathfrak{l} \oplus \mathfrak{m} \cong$ $\mathbb{R} \oplus \mathbb{C}^{n}$. The $\operatorname{Ad}_{S U(n)}$-action on $\mathbb{C}^{n}=\mathbb{R}^{2 n}$ is the usual action and it is trivial on $\mathbb{R}$ (see [39]). Therefore, $\operatorname{ddim} \mathbb{R}[\mathfrak{v}]^{S U(n)}=\operatorname{dind} \mathbb{R}[\mathfrak{v}]^{S U(n)}=2, \mathbb{R}[\mathfrak{v}]^{S U(n)}$ is generated by $h_{0}(x)$ and by a linear function on $\mathfrak{l} \cong \mathbb{R}$, and $c(S U(n+1), S U(n))=0$. Note that $\left(S^{2 n+1}=\right.$ $\left.S U(n+1) / S U(n), d s_{\lambda}^{2}\right)$ can be seen as a distance sphere in a complex projective space $\mathbb{C P}^{n+1}=S U(n+2) / S(U(n+1) \times U(1))($ see $[39,[5])$. 
Example 7: $(G, K, H)=(S p(n+1), S p(1) \times S p(n), U(1) \times S p(n)), G / H=\mathbb{C P}^{2 n+1}$. Here we have a natural inclusion $U(1) \subset S p(1), \operatorname{sp}(1)=u(1) \oplus \mathbb{C}$ and $\mathfrak{l} \cong \mathbb{C}, \mathfrak{m} \cong \mathbb{H}^{n} . U(1)$ acts by a rotation on $\mathbb{C}$ and trivially on $\mathbb{H}^{n}$ and $S p(n)$ acts trivially on $\mathbb{C}$ and by its standard representation on $\mathbb{H}^{n}$. Thus $\operatorname{ddim} \mathbb{R}[\mathfrak{v}]^{S p(n+1)}=\operatorname{dind} \mathbb{R}[\mathfrak{v}]^{S p(n+1)}=2, \mathbb{R}[\mathfrak{v}]^{S p(n+1)}$ is generated by $h_{0}(x)$ and $\delta(x)$, and $c(S p(n+1), U(1) \times S p(n))=0$.

\section{Acknowledgments}

I am greatly thankful to Dmitri Alekseevsky for pointing out the problem of geodesic flows on g.o. spaces and to the referee for useful remarks which helped me to improve the exposition and to extend some results of the paper. This research was supported by the Serbian Ministry of Science, Project 174020 Geometry and Topology of Manifolds, Classical Mechanics and Integrable Dynamical Systems.

\section{References}

[1] Alekseevsky, D. and Arvanitoyeorgos, A.: Riemannian flag manifolds with homogeneous geodesics. Trans. Amer. Math. Soc. 359 (2007), no. 8, 3769-3789

[2] Alekseevsky, D. V. and Nikonorov, Y.G.: Compact Riemannian manifolds with homogeneous geodesics. SIGMA 5 (2009), Paper 093, 16 pp, arXiv:0904.3592 [math.DG]

[3] Arvanitoyeorgos, A., Dzhepko, V. V. and Nikonorov, Yu. G.: Invariant Einstein metrics on some homogeneous spaces of classical Lie groups, Canadian Journal of Mathematics 61 (2009), no. 6, 1201-1213, arXiv: math/0612504 [math.DG].

[4] Arzhantsev, I. V. and Chuvashova, O. V.: Classification of affine homogeneous spaces of complexity one. (Russian) Mat. Sb. 195 (2004), no. 6, 3-20; translation in Sb. Math. 195 (2004), no. 5-6, 765-782

[5] Berndt, J. and Vanhecke, L.: Geometry of weakly symmetric spaces. J. Math. Soc. Japan 48 (1996), no. 4, 745-760.

[6] Berndt, J., Kowalski, O. and Vanhecke, L.: Geodesics in weakly symmetric spaces. Ann. Global Anal. Geom. 15 (1997), no. 2, 153-156.

[7] Besse, A.: Einstein Manifolds, Springer, A Series of Modern Surveys in Mathematics, (1987)

[8] Bolsinov, A. V. and Jovanović, B.: Integrable geodesic flows on homogeneous spaces, Matem. Sbornik 192 (2001), no. 7, 21-40 (Russian); English translation: Sb. Mat. 192 no. 7-8, 951969, (2001)

[9] Bolsinov, A. V. and Jovanović, B.: Non-commutative integrability, moment map and geodesic flows. Annals of Global Analysis and Geometry 23, no. 4, 305-322 (2003), arXiv: math-ph/0109031

[10] Bolsinov, A. V. and Jovanović, B.: Complete involutive algebras of functions on cotangent bundles of homogeneous spaces. Mathematische Zeitschrift 246 no. 1-2, 213-236 (2004)

[11] Brailov, A. V.: Construction of complete integrable geodesic flows on compact symmetric spaces. Izv. Acad. Nauk SSSR, Ser. matem. 50, no.2, 661-674 (1986) (Russian); English translation: Math. USSR-Izv. 50, no.4, 19-31 (1986)

[12] Dragović, V., Gajić, B. and Jovanović, B.: Singular Manakov Flows and Geodesic Flows of Homogeneous Spaces of $S O(n)$, Transfomation Groups 14, no. 3, 513-530 (2009), arXiv: 0901.2444 
[13] Dušek, Z., Kowalski, O. and Nikčević, S.: New examples of Riemannian g.o. manifolds in dimension 7. Differential Geom. Appl. 21 (2004), no. 1, 65-78.

[14] Fedorov, Yu. N. and Jovanović, B.: Geodesic flows and Neumann systems on Stiefel varieties: geometry and integrability, Mathematische Zeitschrift 270(2012), 659-698, arXiv:1011.1835 [nlin.SI]

[15] Gordon, C. S.: Homogeneous Riemannian manifolds whose geodesics are orbits. Topics in geometry, 155-174, Progr. Nonlinear Differential Equations Appl., 20, Birkhuser Boston, Boston, MA, 1996.

[16] Jensen, G.: Einstein metrics on principal fiber bundles, J. Diff. Geom. 8 (1973), 599-614.

[17] Jovanović, B.: Symmetries and integrability. Publ. Inst. Math. (Beograd) (N.S.) 84(98) (2008), 1-36. arXiv:0812.4398 [math.SG]

[18] Jovanović, B.: Integrability of Invariant Geodesic Flows on n-Symmetric Spaces, Annals of Global Analysis and Geometry, 38 (2010), 305-316, arXiv:1006.3693 [math.DG]

[19] Kaplan, A.: On the geometry of groups of Heisenberg type. Bull. London Math. Soc. 15 (1983), no. 1, 35-42.

[20] Kowalski, O. and Vanhecke, L.: Riemannian manifolds with homogeneous geodesics. Boll. Un. Mat. Ital. B (7) 5 (1991), no. 1, 189-246.

[21] Kowalski, O. and Nikčević, S.: On geodesic graphs of Riemannian g.o. spaces. Arch. Math. (Basel) 73 (1999), no. 3, 223-234; Appendix: Arch. Math. (Basel) 79 (2002), no. 2, 158-160.

[22] Lacomba, E. A.: Mechanical systems with symmetry on homogeneous spaces. Trans. Amer. Math. Soc. 185 (1973), 477-491.

[23] Laurent-Gengoux, C., Miranda, E. and Vanhaecke, P.: Action-angle Coordinates for Integrable Systems on Poisson Manifolds, International Mathematics Research Notices, doi:10.1093/imrn/rnq130, arXiv: 0805.1679 [math.SG].

[24] Mishchenko, A. S. and Fomenko, A. T.: Generalized Liouville method of integration of Hamiltonian systems. Funkts. Anal. Prilozh. 12, No.2, 46-56 (1978) (Russian); English translation: Funct. Anal. Appl. 12, 113-121 (1978)

[25] Mikityuk, I. V.: On the integrability of invariant Hamiltonian systems with homogeneous configuration spaces. Matem. Sbornik, 129(171), no.4, 514-534 (1986) (Russian); English translation: Math. USSR Sbornik, 57, no.2, 527-547 (1987)

[26] Mykytyuk, I. V. and Panasyuk, A.: Bi-Poisson structures and integrability of geodesic flows on homogeneous spaces, Transformation Groups 9(3), 289-308, (2004)

[27] Mykytyuk, I. V. and Stepin, A. M.: Classification of almost spherical pairs of compact simple Lie groups. In: Poisson geometry, Banach Center Publ., 51, Polish Acad. Sci., Warsaw, 2000, pp. 231-241.

[28] Mykytyuk, I. V.: Actions of Borel subgroups on homogeneous spaces of reductive complex Lie groups and integrability. Composito Math. 127, 55-67, (2001).

[29] Nekhoroshev, N. N.: Action-angle variables and their generalization. Tr. Mosk. Mat. O.-va. 26, 181-198, (1972) (Russian); English translation: Trans. Mosc. Math. Soc. 26, 180-198 (1972)

[30] Panyushev, D. I.: Complexity of quasiaffine homogeneous varieties, t-decompositions, and affine homogeneous spaces of complexity 1, Adv. in Soviet Math. 8, 151-166 (1992). 
[31] Sadetov, S. T.: A proof of the Mishchenko-Fomenko conjecture (1981). Dokl. Akad. Nauk 397, no. 6, 751-754 (2004) (Russian)

[32] Selberg, A.: Harmonic analysis and discontinuous groups in weakly symmetric Riemannian spaces with applications to Dirichlet series. J. Indian Math. Soc. (N.S.) 20 (1956), 47-87.

[33] Tamaru, H.: Riemannian geodesic orbit metrics on fiber bundles. Algebras Groups Geom. 15 (1998), no. 1, 55-67.

[34] Tamaru, H.: Riemannian G.O. spaces fibered over irreducible symmetric spaces. Osaka J. Math. 36 (1999), no. 4, 835-851.

[35] Thimm A.: Integrable geodesic flows on homogeneous spaces, Ergod. Th. \& Dynam. Sys.,1, 495-517 (1981)

[36] Tóth, G. Z.: On Lagrangian and Hamiltonian systems with homogeneous trajectories, J. Phys. A: Math. Theor. 43 (2010) 385206, arXiv: 1003.1495v3 [math-ph]

[37] Vinberg, E. B.: Commutative homogeneous spaces and co-isotropic symplectic actions. Uspekhi Mat. Nauk 56, No. 1, 3-62 (2001) (Russian); English translation: Russian Math. Surveys 56, No. 1, 1-60 (2001)

[38] Yakimova, O. S. Weakly symmetric Riemannian manifolds with a reductive isometry group. (Russian) Mat. Sb. 195 (2004), no. 4, 143-160; translation in Sb. Math. 195 (2004), no. 3-4, 599-614

[39] Ziller, W.: Homogeneous Einstein metrics on Spheres and Projective Spaces, Math. Ann. 259 (1982) 351-358.

[40] Ziller, W.: Weakly symmetric spaces. Topics in geometry, 355-368, Progr. Nonlinear Differential Equations Appl., 20, Birkhuser Boston, Boston, MA, 1996. 